\title{
Metabolome-guided genomics to identify pathogenic variants in isocitrate dehydrogenase, fumarate hydratase, and succinate dehydrogenase genes in pheochromocytoma and paraganglioma
}

\author{
Susan Richter, $\mathrm{PhD}^{1}$, Laura Gieldon, $\mathrm{MD}^{2}$, Ying Pang, $\mathrm{MD} \mathrm{PhD}^{3}$, Mirko Peitzsch, $\mathrm{PhD}^{1}$, \\ Thanh Huynh, $\mathrm{BSc}^{3}$, Rocio Leton, $\mathrm{BSc}^{4}$, Bruna Viana, $\mathrm{BSc}^{3}$, Tonino Ercolino, $\mathrm{PhD}^{5}$, \\ Anastasios Mangelis, $\mathrm{MSc}^{1}$, Elena Rapizzi, $\mathrm{PhD}^{6}$, Mario Menschikowski, PhD ${ }^{1}$, Daniela Aust, $\mathrm{MD}^{7}$, \\ Matthias Kroiss, MD PhD ${ }^{8}$, Felix Beuschlein, MD ${ }^{9,10}$, Volker Gudziol, MD ${ }^{11}$, \\ Henri JLM Timmers, MD, PhD ${ }^{12}$, Jacques Lenders, MD, PhD ${ }^{12,13}$, Massimo Mannelli, MD ${ }^{6}$, \\ Alberto Cascon, $\mathrm{PhD}^{4}$, Karel Pacak, $\mathrm{MD}, \mathrm{PhD}^{3}$, Mercedes Robledo, $\mathrm{PhD}^{4}$, \\ Graeme Eisenhofer, PhD ${ }^{1,13}$ and Barbara Klink, MD ${ }^{2}$
}

\begin{abstract}
Purpose: Metabolic aberrations have been described in neoplasms with pathogenic variants (PV) in the Krebs cycle genes encoding succinate dehydrogenase (SDH), fumarate hydratase (FH) and isocitrate dehydrogenase (IDH). In turn, accumulation of oncometabolites succinate, fumarate, and 2-hydroxyglutarate can be employed to identify tumors with those PV . Additionally, such metabolic readouts may aid in genetic variant interpretation and improve diagnostics.
\end{abstract}

Methods: Using liquid chromatography-mass spectrometry, 395 pheochromocytomas and paragangliomas (PPGLs) from 391 patients were screened for metabolites to indicate Krebs cycle aberrations. Multigene panel sequencing was applied to detect driver PV in cases with indicative metabolite profiles but undetermined genetic drivers.

Results: Aberrant Krebs cycle metabolomes identified rare cases of PPGLs with germline PV in $F H$ and somatic PV in $I D H x$ and SDHx, including the first case of a somatic IDH2 PV in PPGL.
Metabolomics also reliably identified PPGLs with SDHx loss-offunction (LOF) PV. Therefore we utilized tumor metabolite profiles to further classify variants of unknown significance in $S D H x$, thereby enabling missense variants associated with $S D H x$ LOF to be distinguished from benign variants.

Conclusion: We propose incorporation of metabolome data into the diagnostics algorithm in PPGLs to guide genetic testing and variant interpretation and to help identify rare cases with $\mathrm{PV}$ in $\mathrm{FH}$ and $\mathrm{IDH}$.

Genetics in Medicine (2019) 21:705-717; https://doi.org/10.1038/s41436018-0106-5

Keywords: next-generation sequencing; variant of unknown significance; succinate; fumarate; 2-hydroxyglutarate

\section{INTRODUCTION}

Pathogenic variants (PV) in Krebs cycle-related genes occur in different tumor entities, which hence share a common mechanism of tumorigenesis and might also respond to similar treatment strategies. ${ }^{1,2}$ Currently, there are nine genes encoding several different Krebs cycle enzymes indicated as tumor drivers: fumarate hydratase $(F H) \mathrm{PV}$ are associated with renal cell carcinomas (RCC), leiomyomas, and pheochromocytomas and paragangliomas (PPGLs). ${ }^{3,4}$ In isocitrate dehydrogenase genes 1 or 2 (IDH1/2) occur in gliomas, acute myeloid leukemia, cholangiocarcinomas, and rarely also in PPGL $^{5-8} \mathrm{PV}$ in any of the five succinate dehydrogenase subunits $(S D H x)$ or accessory proteins can lead to gastrointestinal stromal tumors, RCC, PPGL, neuroblastoma, and

\footnotetext{
${ }^{1}$ Institute of Clinical Chemistry and Laboratory Medicine, University Hospital Carl Gustav Carus, Medical Faculty Carl Gustav Carus, Technische Universität Dresden, Dresden, Germany; ${ }^{2}$ Institute for Clinical Genetics, Medical Faculty Carl Gustav Carus, Technische Universität Dresden, Dresden, Germany; ${ }^{3}$ Eunice Kennedy Shriver National Institute of Child Health and Human Development, National Institutes of Health, Bethesda, Maryland, USA; ${ }^{4}$ Hereditary Endocrine Cancer Group, CNIO, Madrid, Spain and Centro de Investigación Biomédica en Red de Enfermedades Raras (CIBERER), Madrid, Spain; ${ }^{5}$ Azienda Ospedaliero-Universitaria Careggi, Endocrinology Unit, Florence, Italy; ${ }^{6}$ Department of Experimental and Clinical Medicine, University of Florence, Florence, Italy; ${ }^{7}$ Institute of Pathology, Tumor and Normal Tissue Bank of the UCC/NCT Dresden, University Hospital Carl Gustav Carus, Technische Universität Dresden, Dresden, Germany; ${ }^{8}$ Department of Internal Medicine, Division of Endocrinology, University Hospital, University of Würzburg, Würzburg, Germany; ${ }^{9}$ Medizinische Klinik and Poliklinik IV, Ludwig-Maximilians-Universität München, Munich, Germany; ${ }^{10}$ Department for Endocrinology, Diabetology and Clinical Nutrition, UniversitätsSpital Zürich, Zurich, Switzerland; ${ }^{11}$ Departments of Otorhinolaryngology, University Hospital Carl Gustav Carus, Technische Universität Dresden, Dresden, Germany; ${ }^{12}$ Department of Internal Medicine, Radboud University Medical Centre, Nijmegen, The Netherlands; ${ }^{13}$ Department of Medicine III, University Hospital Dresden, Dresden, Germany. Correspondence: Susan Richter (Susan.Richter2@uniklinikum-dresden.de)
} 
pituitary tumors. ${ }^{9-11}$ Additionally, malate dehydrogenase 2 (MDH2) was found to be rarely mutated in PPGL. ${ }^{12}$

In PPGLs, catecholamine-producing tumors originating from adrenal or extra-adrenal chromaffin tissue, tumor development has been ascribed to $\mathrm{PV}$ in more than 20 susceptibility genes, with $30-40 \%$ of PPGLs having a hereditary background. ${ }^{13}$ Identification of tumors due to SDHX and FH PV has implications for patient care and follow-up, since those patients are at higher risk for metastatic disease. ${ }^{3,14}$ Moreover, distinguishing between sporadic tumors and cases with underlying germlinen PV is important for management of patients and their families.

$S D H x-, F H-$, and $I D H 1 / 2$-mutated tumors are known to lead to abnormally high levels of one of several oncometabolites: succinate, fumarate, or 2-hydroxyglutarate (2HG). Increases in those result in the inhibition of a-ketoglutaratedependent enzymes, including prolyl hydroxylases and DNA and histone demethylases, causing a pseudohypoxic and hypermethylator phenotype. ${ }^{15} \mathrm{We}$ and others have already established the succinate:fumarate ratio as a diagnostically highly accurate functional indicator of pathogenic SDHx variants. ${ }^{16,17}$ Strong elevations of fumarate as a consequence of loss of function (LOF) PV in FH are well known from RCC and leiomyomas, and have also been described for PPGL. 3,18 Somatic hotspot PV of codons R132 and R172 within IDH1 and $I D H 2$, respectively, lead to a gain of function responsible for the production of the D-enantiomer of $2 \mathrm{HG}, \mathrm{D}-2 \mathrm{HG}{ }^{19}$ Measurement of metabolites can therefore provide a screening tool to identify tumors with underlying driver PV in those genes. On the other hand, metabolomics could also function as readout for classification of identified variants in metabolic enzymes.

Clinical practice guidelines recommend that genetic testing should be considered in all patients with PPGL, with testing and test interpretation carried out according to a clinical feature-driven diagnostic algorithm that establishes the likelihood of PV. ${ }^{20}$ With the use of next-generation sequencing (NGS) now moving into routine clinical diagnostics, large numbers of genes can be tested simultaneously as part of one panel. ${ }^{13,21-23}$ As a consequence, more variants are identified; however, for many the pathogenicity is unclear and caution is warranted for interpretation, in particular for germline variants with far-reaching implications for patients and their families. $^{21,22,24}$

The importance of correct classification of gene variants is emphasized by the example of RET Y791F, a variant previously associated with the occurrence of medullary thyroid carcinoma. Recent findings, however, showed that this variant is likely benign and may have led to unnecessary prophylactic thyroidectomies. ${ }^{25}$ The development of standards and guidelines for the correct interpretation of genetic variants and the need for new approaches to functionally assess variants of unknown significance (VUS) has become increasingly significant. ${ }^{26}$ Apart from manipulated cell lines and in silico predictions, potential approaches to assess functionality include immunohistochemistry (IHC), ${ }^{10}$ expression profiling ${ }^{27}$ as well as nuclear magnetic resonance, ${ }^{28}$ and mass spectrometry metabolite profiling. ${ }^{16,17}$

The present study utilized mass spectrometry-based metabolomics of Krebs cycle intermediates in 395 PPGL samples to identify aberrant metabolite profiles, functionally relate them to variants in relevant genes, and evaluate their potential as a diagnostic tool.

\section{MATERIALS AND METHODS}

\section{Tumor procurement}

Primary tumor tissue was collected from 391 patients with PPGL from nine centers, specimens either snap frozen or formalin-fixed and paraffin-embedded (FFPE) after surgery (Supplementary information 1). Tumor procurement was approved under Intramural Review Board protocols at each center, where patients have signed informed consent. Samples with potential contaminants (e.g., those stored in RNApreserving citrate-based buffers) were excluded from metabolic clustering analysis. We included 230 tumors from 229 patients from a previous study that analyzed succinate: fumarate in relation to $S D H x \mathrm{PV}^{16}$ and 157 freshly frozen (FF) tumors from 154 newly diagnosed patients from seven centers (Bethesda/USA, Madrid/Spain, Florence/Italy, Nijmegen/The Netherlands, Dresden, Munich, Würzburg/Germany). Additionally, eight patients in whom only FFPE tissue was available were included.

\section{Krebs cycle metabolites}

In a mass spectrometry-based screening assay eight organic acids of the Krebs cycle as well as pyruvate and lactate were measured in all 387 FF and 8 FFPE tissue samples. Additionally, four amino acids, glutamate, glutamine, aspartate, and asparagine, were analyzed in a sample subset. Metabolites were analyzed with a modification of a previously described method. ${ }^{16}$ Briefly, metabolites were extracted from samples with methanol, dried, resuspended in mobile phase, and cleared with a $0.2-\mu \mathrm{m}$ centrifugal filter. To improve separation, the elution gradient was changed as follows: $99 \%$ A $(0.2 \%$ formic acid in water), $1 \%$ B $(0.2 \%$ formic acid in acetonitrile) for $2.00 \mathrm{~min}, 100 \% \mathrm{~B}$ at 2.50 to $2.65 \mathrm{~min}, 1 \% \mathrm{~B}$ at $3.40 \mathrm{~min}$, and equilibration with $1 \% \mathrm{~B}$ until $5.00 \mathrm{~min}$. Multiple reaction monitoring with negative electrospray ionization was used for quantification according to transitions provided in Supplementary Table S1.

\section{D-/L-Enantiomers of 2-hydroxyglutarate (2HG)}

Samples were prepared as described previously. ${ }^{29}$ Briefly, methanol extracts of tumor tissue were dried and derivatized using diacetyl-1-tartaric anhydride (DATAN) dissolved in dichloromethane and acetic acid (4:1). After 30 min incubation at $75^{\circ} \mathrm{C}$, samples were dried under a stream of nitrogen. After reconstitution in mobile phase $(3.5 \%$ acetonitrile in water containing $2 \mathrm{mM}$ ammonium formate, $\mathrm{pH}$ 3.6), samples were injected onto a Shimadzu UFLC LC-20ADXR-system coupled to a tandem mass spectrometer (Sciex QTRAP5500). Separation of D- and L-enantiomer derivatives of $2 \mathrm{HG}$ was 
achieved using a Waters XBridge ${ }^{\circledR}$ BEH C18 column $(100 \times 3$ $\mathrm{mm}, 2.5 \mu \mathrm{m}$ ) with an isocratic mobile phase flow at $0.44 \mathrm{ml} /$ min.

\section{Genetic testing}

Patients were tested for germline PV in established susceptibility genes by centers of origin or by the Spanish National Cancer Research Centre (CNIO) institute in Madrid through a collaborative multicenter study (the Prospective Monoamine-producing Tumor Study, https://pmt-study. pressor.org/) using Sanger sequencing and/or NGS, and multiplex ligation-dependent probe amplification or custom array comparative genomic hybridization (CGH) for deletion detection.

Tumors with aberrant metabolite profiles and unknown genetic backgrounds were additionally analyzed by customized panel sequencing (Illumina, San Diego, CA) including coding exons and $20 \mathrm{bp}$ of intronic flanking regions of known PPGL-associated genes RET, VHL, NF1, MAX, TMEM127, SDHA, SDHB, SDHC, SDHD, SDHAF2, MDH2, FH, EPAS1, HRAS, KIF1B, EGLN1, EGNL2, IDH1, and IDH2. Library preparation was performed using Illuminas' TruSeq Nano DNA Library Prep Kit according to the manufacturer's instructions. One hundred fifty nt paired-end sequencing was carried out with a minimum median coverage of 1000 -fold either on an Illumina MiSeq or NextSeq sequencer. Reads were aligned to the reference genome (GRCh37/hg19) and variant calling was performed using in-house workflows, as described previously. ${ }^{22,30}$ Variants of interest were validated by Sanger sequencing.

Variant classification was performed in accordance with the standards and guidelines of the American College of Medical Genetics and Genomics and the Association for Molecular Pathology (ACMG-AMP), ${ }^{26}$ considering loss of heterozygosity (LOH) in tumor samples as an additional supporting criterion for pathogenicity (PP6). For $S D H x$ variants, immunohistochemical loss of the SDH complex as well as an elevated succinate:fumarate were interpreted as functional analyses (PS3) but were downgraded to moderate (PM7 and PM8) criteria. In contrast, positive immunohistochemistry of the SDH complex and normal succinate:fumarate were interpreted to support nonfunctional variants (BP8).

\section{Immunohistochemistry}

FFPE tumor tissue was sectioned and stained for SDHB or $\mathrm{FH}$ protein using rabbit polyclonal anti-SDHB (HPA002868, Sigma-Aldrich), anti-SDHA (ab14715, Abcam or 2E3GC12FB2AE2, Biorad), and anti-FH (HPA027341, Sigma-Aldrich).

\section{Statistical analysis}

Metabolome data were normalized to natural logarithms (LN) to account for large value discrepancies. Basic statistics and clustering analysis were performed using JMP statistics software (version Pro 12.1.0). Outlier analysis on LNtransformed values or ratios were used to identify candidates for panel sequencing of metabolic genes. For this purpose we focused on outliers above the 75th quartile $+1.5 \times$ (interquartile range) marked by the top whisker in box and whisker plots. Significance between groups of patients was calculated using the nonparametric Wilcoxon test.

\section{Metabolite profiling in PPGL}

\section{RESULTS}

Clustering analysis of the full set of 395 PPGLs from 391 patients using ratios of Krebs cycle intermediates identified two clusters involving three main groups (Fig. 1a, Supplementary information 1): cluster I was characterized by high succinate and low levels of all other Krebs cycle metabolites and almost exclusively contained SDHx-mutated tumors. Additionally, lower levels of the amino acids aspartate and glutamate were present in $S D H x$-mutated PPGLs compared with PPGLs due to other causes (Supplementary Fig. S1). PPGLs of the first cluster, with the exception of three tumors, had succinate:fumarate ratios above a previously defined cutoff for SDHx-mutated PPGLs (Fig. 1b). ${ }^{16}$

Cluster II was divided into two main subclusters separated by different levels of citrate, isocitrate, and cis-aconitate (Fig. 1a). Subcluster IIa was characterized by low levels of the aforementioned metabolites and contained primarily tumors with driver $\mathrm{PV}$ ns in the hypoxia signaling axis (VHL, EPAS1), as well as tumors with $F H$ and IDHx PV (characterized below in detail). Subcluster IIb, characterized by higher citrate, isocitrate, and cis-aconitate levels, includes predominantly tumors with driver PV in the kinase signaling pathway (RET, NF1, TMEM127). This metabolic differentiation between groups with PV in common pathways reflects somewhat the clustering of PPGLs according to transcriptomics. ${ }^{31}$

By applying outlier analysis to our cohort, we identified outliers for $2 \mathrm{HG}$ (Fig. 1c), $\alpha$-ketoglutarate (Supplementary Fig. S2A), the fumarate:malate ratio (Fig. 1d), fumarate (Supplementary Fig. S2B), and malate (Supplementary Fig. $\mathrm{S} 2 \mathrm{C})$. The following sections describe the identification of underlying PV in those samples.

\section{HG and $\alpha$-ketoglutarate elevations and IDHx PV in PPGL}

Twelve outliers were identified for $2 \mathrm{HG}$. Of those, two were also a-ketoglutarate outliers (Fig. 1c, Supplementary Fig. S1A). Nine of the twelve $2 \mathrm{HG}$ outliers were available for further analysis. Patients 1 and 2 had the highest $2 \mathrm{HG}$ values of the set, with increases of up to 1800 -fold (patient 2) and 100-fold (patient 1) over the median (Fig. 2a). The other seven tumors showed increases of 10 - to 40 -fold above the median and four of nine PPGLs also had increases in $a$ ketoglutarate of 9- to 48-fold over the median (Fig. 2b).

To further characterize the $2 \mathrm{HG}$ elevations, $\mathrm{D}$ - and Lenantiomers of $2 \mathrm{HG}$ were measured. Seven PPGLs with moderate total $2 \mathrm{HG}$ levels served as controls with D-2HG to L-2HG ratios ranging from 0.2 to 1.6 (Fig. 2c). Tumors of patients 1 and 2 showed strongly increased D-2HG to L-2HG ratios of 127 and 901, respectively (Fig. 2d). Tumors from 
patients 3 and 4 had L-2HG to D-2HG ratios of 13 with control tumors ranging from 0.4 to 4.3. The other five PPGLs were similar to controls.
Patient 1 has been described elsewhere with a somatic $I D H 1$ c.394C > T (R132C) PV, ${ }^{6}$ explaining the strong increases in D$2 \mathrm{HG}$ now described here for the first time. In patient 2 we a

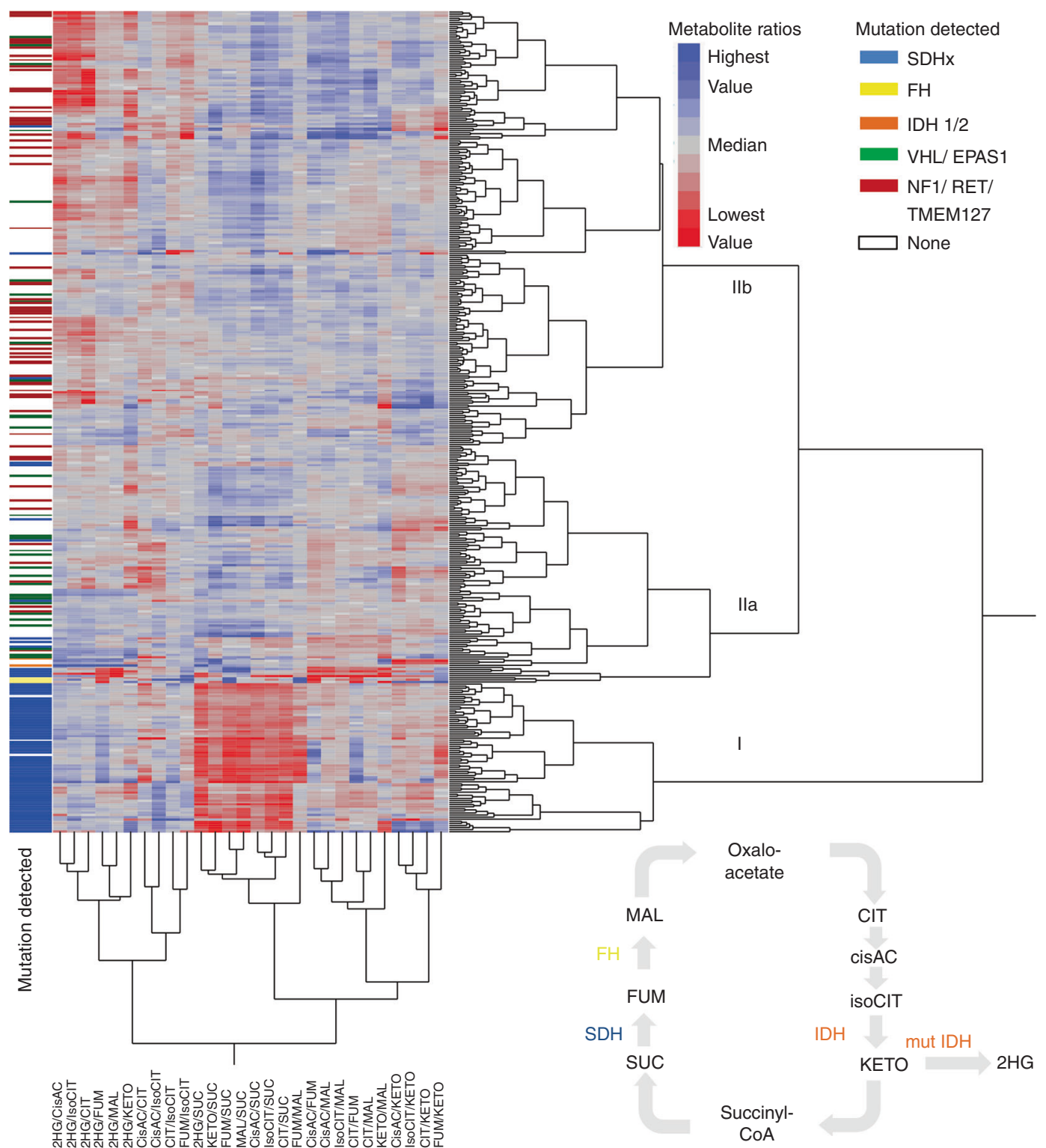

b

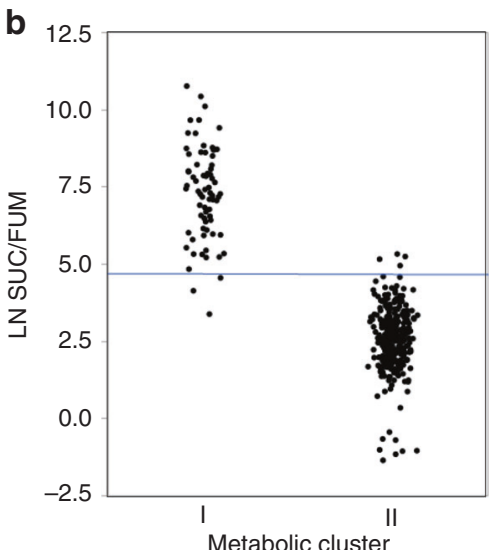

C

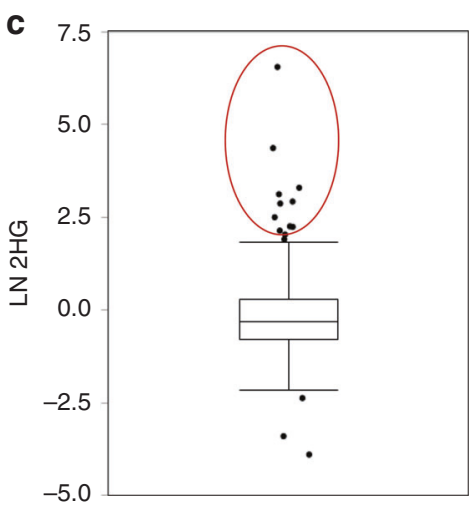

d

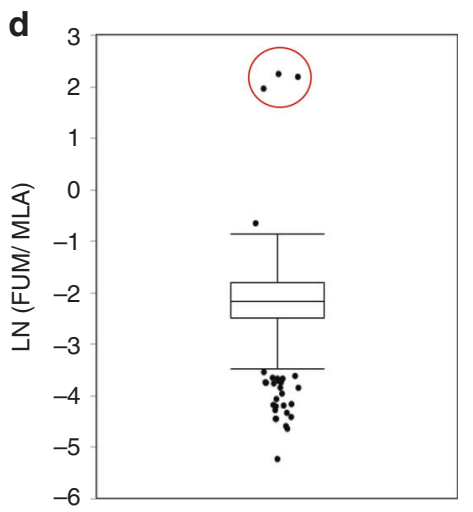


Fig. 1 Krebs cycle metabolite profiling of pheochromocytomas and paragangliomas (PPGLs). a Hierarchical cluster analysis (Ward) of 395 different PPGLs from 391 patients. All possible ratios between eight intermediates (citrate, isocitrate, cis-aconitate, $\alpha$-ketoglutarate, 2-hydroxyglutarate, succinate, fumarate, malate) of the central carbon metabolism were generated and natural logarithm (LN) transformed. Mutational status (germline and somatic) is shown by color in the left column. Clusters are marked with roman numbers. The Krebs cycle with all measured metabolites is schematically shown; oxaloacetate and succinyl-CoA are not detectable with the used method. 2HG-2 hydroxyglutarate, CIT citrate, cisAC cis-aconitate, FUM fumarate, isoCIT isocitrate, KETO $\alpha$-ketoglutarate, MAL malate, SUC succinate. b The succinate:fumarate ratio as an indicator for PV in SDHx genes separates cluster I from II. Cut-off defined in ref. ${ }^{16}$ marked in blue. c Box plots of 2 HG levels with outliers marked as dots. Outliers above the top whisker (the 75 th quartile $+1.5 x$ [interquartile range]) were further investigated by next-generation sequencing analysis when the driver PV was unknown (red circle). $\mathbf{d}$ Outlier analysis for the fumarate:malate ratio

identified a somatic IDH2 c.514A>G (R172G) PV, to our knowledge the first $I D H 2$ described in PPGL to date (Fig. 2e). A detailed patient description is provided in Supplementary information 2. The other seven PPGLs with moderately elevated 2HG levels and normal D-2HG to L-2HG ratios did not have PV in any of the genes of our NGS panel; however, in five PPGLs we identified PV in other known susceptibility genes, including NF1, HRAS, ATRX, and VHL (Fig. 2f). In summary, IDH1/2-mutated PPGLs were characterized by elevations of total $2 \mathrm{HG}$ of at least 100 -fold and of D-2HG to L-2HG ratios over 100 -fold above control median values.

\section{Fumarate and malate elevations and FH PV in PPGL}

Outlier analysis identified three samples (patients 10, 11, 12) with strong elevations in fumarate:malate ratios 60- to 80-fold above median values of controls (Fig. 1d). Five outliers for absolute fumarate levels were identified. Three of those also presented as outliers for malate and had normal fumarate: malate ratios (Figs. S2B-C); the other two (patients 10, 11) were also fumarate:malate outliers.

In all three patients with aberrant tumor fumarate:malate, NGS revealed heterozygous germline variants in $F H$ : NM_000143.3:c.700A $>$ G p.(Thr234Ala), c.908T >C p. (Leu303Ser), and c.816_836del p.(Ala273_Val279del) (Figs. 3a-c). In all cases, the tumors showed loss of heterozygosity ( $\mathrm{LOH})$ for these variants. IHC confirmed the loss of FH protein in tumors 10 and 11 , for tumor 12 no more tissue was available (Fig. 3d).

The $F H$ variants in patients 10 and $11-\mathrm{p}$.(Thr234Ala) and p.(Leu303Ser) - are both missense variants predicted to be damaging by three different in silico tools (Polyphen2/ SIFT/MutationTaster). Both are listed three times in heterozygous states in the gnomAD database. FH p.(Thr234Ala) is rated with pathogenic/uncertain significance in ClinVar and dbSNP (rs372505976) and $F H$ p.(Leu303Ser) as a VUS (rs201502246). The variant $F H$ p.(Leu303Ser) was found in a girl together with a second compound heterozygous variant in $F H$, who suffered from autosomal-recessive fumarase deficiency (LOVD 0000116260). ${ }^{32}$ The variant p.(Thr234Ala) has not, to the best of our knowledge, been reported in the literature. The third patient (patient 12) carried an in-frame deletion leading to loss of six amino acids in $F H$ (p.[Ala273_Val279del]). This variant is rated pathogenic in ClinVar and dbSNP (rs863223985). It is not listed in gnomAD or described in the literature. With only this information available, the variants would be interpreted as VUS according to ACMG-AMP criteria. The metabolomicsbased aberrant tumor fumarate:malate ratios and elevated fumarate levels, which indicate loss of function of $F H$, combined with the IHC-confirmed loss of tumor FH protein in patients 10 and 11 and $\mathrm{LOH}$, provides sufficient evidence to classify all three variants as likely pathogenic.

All three patients with germline FH PV (patients 10-12) had unilateral adrenal pheochromocytomas with a noradrenergic biochemical phenotype (see Supplementary information 2). None of the patients had metastatic disease at initial presentation (patient 10) or 6 (patient 11) to 18 years (patient 12) later. Family history included RCC in the father of patient 11 and thyroid cancer and melanoma in patient 10.

The three other fumarate outliers with normal fumarate: malate ratios, who also showed outlier profiles for malate (Supplementary Fig. S2C), did not show variants in $F H$ but had $S D H x$ variants classified as likely pathogenic or pathogenic (Table 1 and Supplementary material 3). Because those tumors did not show aberrant fumarate:succinate ratios, this might indicate that the outlier profiles for those samples are possibly related to sampling problems. In summary, fumarate:malate ratios above 7.0 with non- $F H$ mutated PPGLs having values below 1.0 provide a useful guide to the presence of $F H$ PV in PPGL. Increases in tumoral fumarate alone may not offer reliable guidance to the presence of FH PV.

\section{Succinate:fumarate ratios, metabolic cluster 1, and $S D H x$ variants in PPGL}

In our cohort of 391 patients we identified 73 individuals having PPGLs with aberrant succinate:fumarate ratios. ${ }^{16}$ All except five tumors belonged to the first metabolic cluster (Fig. 1a). Of those 73 patients, 34 had clear LOF PV (e.g., stop gain, frameshift, or exon-spanning deletions) in one of the $S D H x$ genes in the germline. Furthermore, another 32 patients had missense SDHx variants in the germline that are well established as pathogenic or likely pathogenic $(n=21)$ or would be currently classified as VUS $(n=10)$ or likely benign (1), according to ACMG-AMP guidelines (see Tables $\mathbf{1}$ and $\mathbf{2}$ and Supplementary material 3). Additionally, three patients $(57,77,92)$ had tumors of metabolic cluster I with confirmed pathogenic or likely pathogenic SDHx but with succinate: fumarate ratios below the cut-off.

In 7 of the 73 patients with elevated succinate:fumarate ratios, no $S D H x$ variants were identified in the germline. In 
a

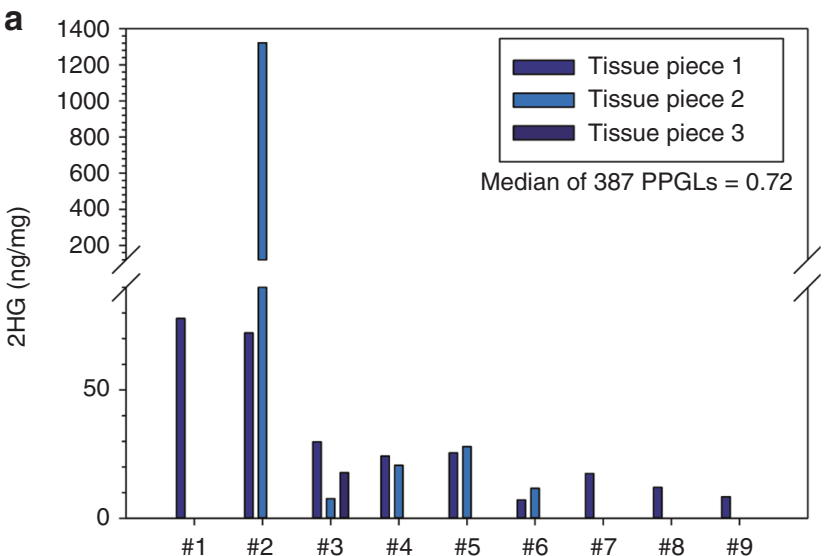

b

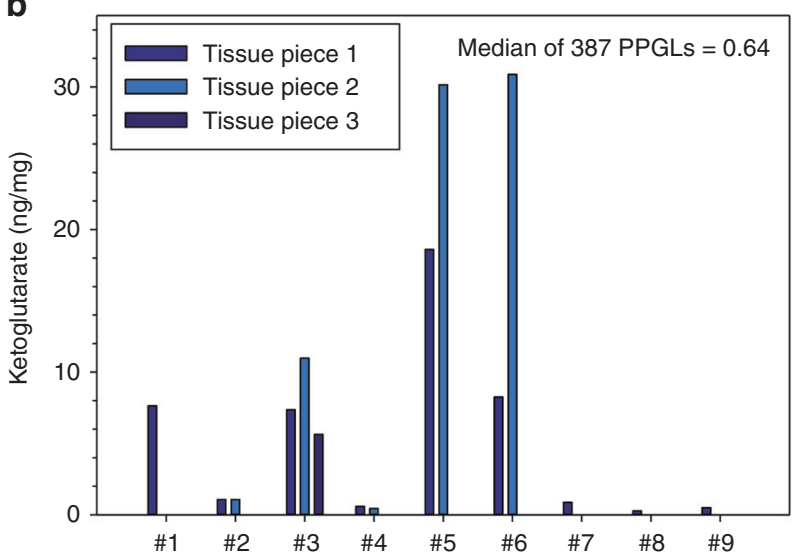

C

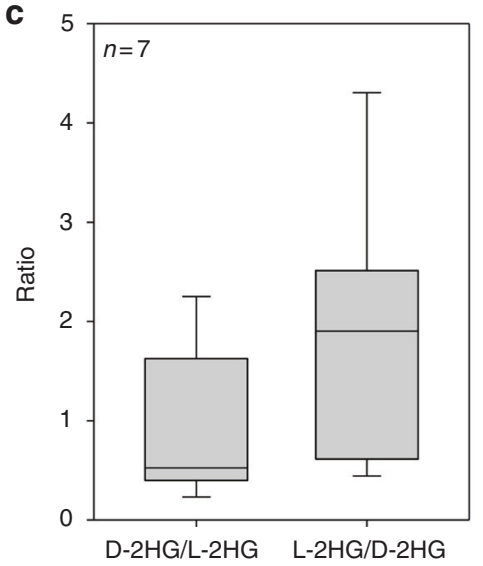

d

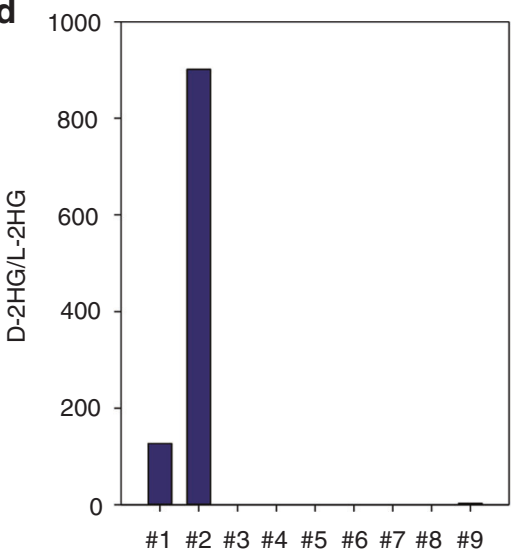

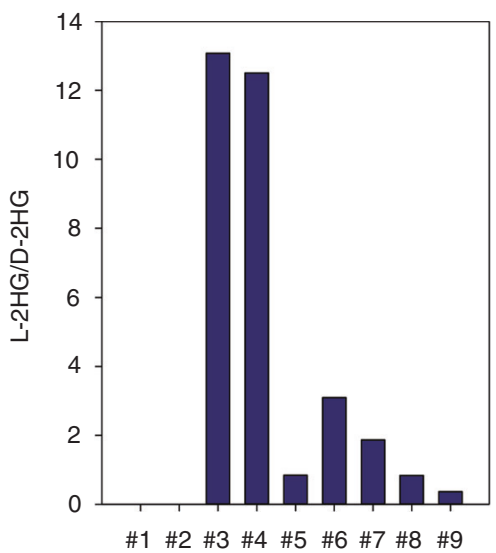

$\mathbf{e}$

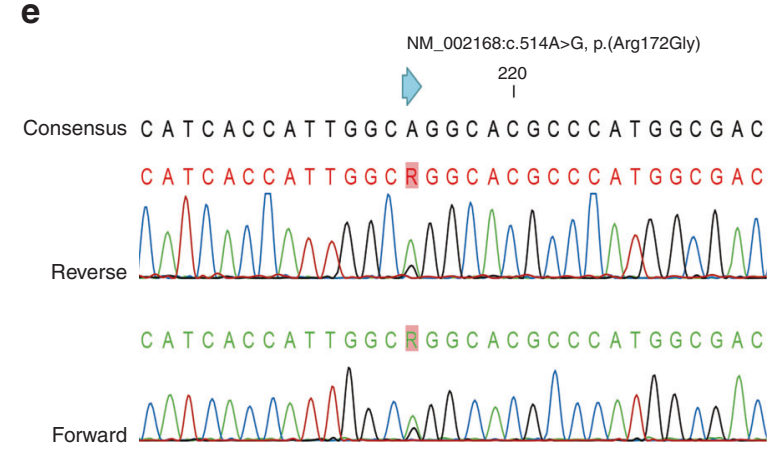

f

\begin{tabular}{cccccc}
\hline$\#$ & $\begin{array}{c}\text { Pathogenic } \\
\text { mutations }\end{array}$ & $\begin{array}{c}\text { Age at } \\
\text { diagnosis }\end{array}$ & Gender & $\begin{array}{c}\text { Tumor } \\
\text { location }\end{array}$ & $\begin{array}{c}\text { Tumor } \\
\text { catechol- } \\
\text { amines }\end{array}$ \\
\hline 1 & IDH1 (s) & 78 & M & PGL & nd \\
2 & IDH2 (s) & 48 & F & HNP & D \\
3 & no & 53 & F & PHEO & N \\
4 & NF1 (s) & 56 & F & PHEO & A \\
5 & no & 48 & M & PGL & N \\
6 & HRAS (s) & 27 & F & PHEO & N \\
7 & VHL (s) & 47 & F & PHEO & nd \\
8 & NF1 (g) & 58 & F & PHEO & nd \\
9 & ATRX (s) & 65 & M & PHEO & nd \\
\hline
\end{tabular}

Fig. 2 Isocitrate dehydrogenase 1 and 2 gain-of-function PV in pheochromocytomas and paragangliomas (PPGLs). a Total 2-hydroxyglutarate $(2 \mathrm{HG})$ in nine tumors with the highest $2 \mathrm{HG}$ values of the set of fresh frozen tumors. $\mathbf{b}$ Total $\alpha$-ketoglutarate in nine tumors with the highest $2 \mathrm{HG}$ values of the set. (c) Quantification of D- and L-enantiomers of 2HG in seven control PPGLs with moderate total 2HG. d Quantification of D-2HG and L-2HG in the nine tumors with highest total $2 \mathrm{HG}$. e Sanger sequencing traces of PPGL 2 analyzed for a PV in IDH2. $\mathbf{f}$ Patient information of PPGLs with highest total $2 \mathrm{HG}$. For further details refer to Supplementary information 1. A adrenergic, D dopaminergic, $g$ germline, HNP head and neck, $N$ noradrenergic, nd not determined, PGL extra-adrenal, PHEO adrenal, s somatic variant, \# patient number

three of those patients we identified somatic $S D H x$ variants: a stop PV (SDHB p.Tyr61*, 30); a startlossPV (SDHD c.3G $>\mathrm{T}$, 70), and a missense PV (SDHB p.(Pro155Arg), 105) (Supplementary material 3 ). In a further patient we previously reported somatic SDHC-promoter methylation (107 [ref. ${ }^{33}$ ]) explaining the SDH-deficiency phenotype. Two cases (106, 108) currently remain unsolved. We excluded somatic $S D H x$ PV and SDHC-promoter methylation in those cases. In 106 we reported germline variants in OGDHL and PCK2; however their significance is unclear. ${ }^{6}$ The seventh case had a pathogenic germline RET c.1852T>G, p.(Cys618Gly) PV. Therefore, we interpreted the borderline succinate:fumarate elevation (109) as a false-positive result.

One patient (69) with an elevated tumoral succinate: fumarate was particularly interesting. He had a likely benign germline missense variant SDHD c.34G>A, p.(Gly12Ser), 
a

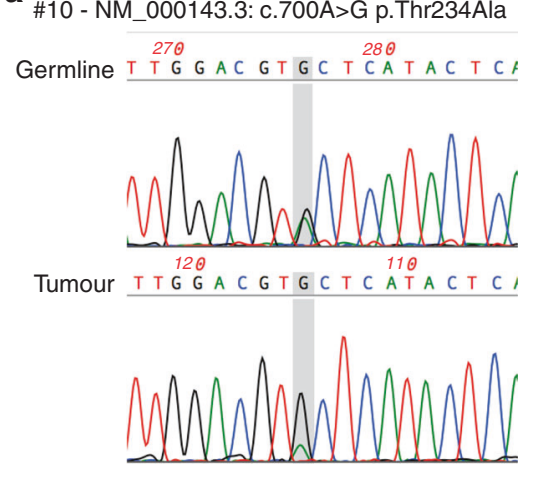

C
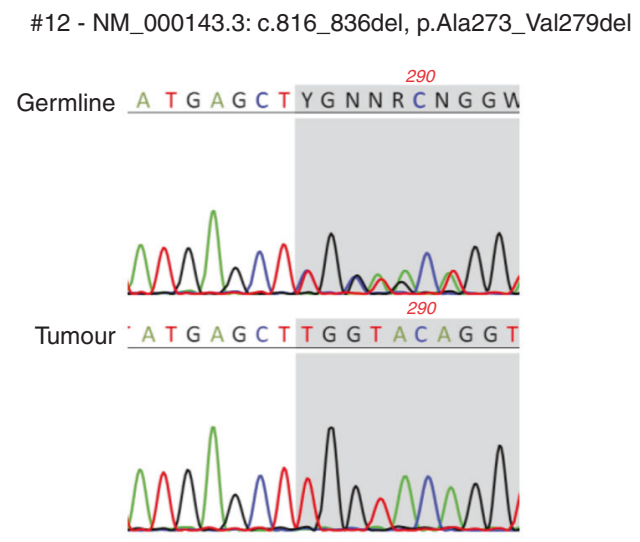

b

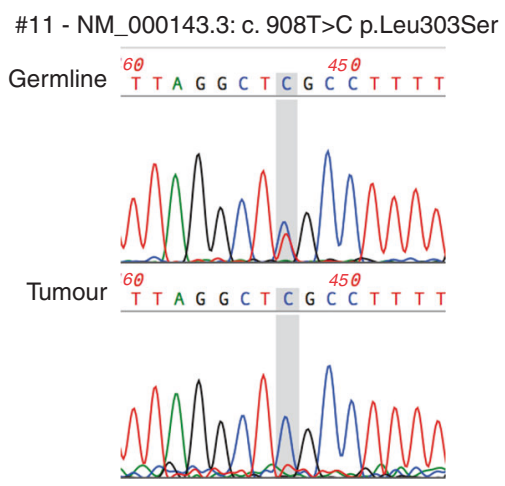

d

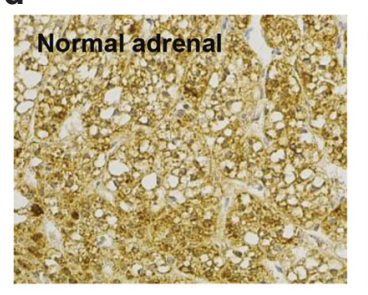

$\# 10$
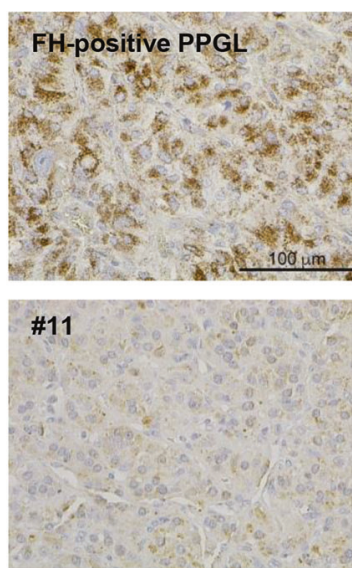

Fig. 3 Metabolite profiling identifies pathogenic fumarate hydratase (FH) PV previously classified as variants of unknown significance (VUS). Sanger sequencing traces of leukocyte (germline) and tumor DNA from a patient 10, b patient 11 and $\mathbf{c}$ patient 12. d Immunohistochemical staining for FH of normal adrenal, FH wild type, and FH mutated pheochromocytoma and paraganglioma (PPGL) tissue from patients 10 and 11; 400x magnification

which is listed 2035x heterozygously and 12x homozygously in the gnomAD database and has been described as a rare polymorphism. ${ }^{34}$ The patient presented with sympathetic paraganglioma of the renal hilum at age 21 and had no family history of PPGL. Because germline testing was inconclusive in this case, we analyzed tumor tissue and identified a pathogenic somatic LOF variant in SDHD NM_003002.3: c.337_340del, p.(Asp113Metfs $\left.{ }^{\star} 21\right)$ in $41.47 \%$ of reads. Allele frequency of the germline variant c.34G>A was $69.7 \%$ in the tumor, indicating $\mathrm{LOH}$ of the wild-type $S D H D$ allele and that the somatic and pathogenic SDHD variant p.(Asp113Metfs $\left.{ }^{\star} 21\right)$ occurred in the allele having the germline c. $34 \mathrm{G}>\mathrm{A}$ variant. We therefore assume that the somatic $S D H D$ variant p.(Asp113Metfs ${ }^{\star 21}$ ) is responsible for $\mathrm{SDH}$ deficiency in this tumor and that the germline variant is likely benign and coincidentally observed together with the aberrant metabolite profile.

In ten patients with elevated succinate:fumarate ratios we identified eight different missense variants in $S D H x$ (including one somatic) that would currently be classified as VUS. Most of the variants had previously been described in PPGL cases and integrating the metabolomics profiles together with all available information (see Supplementary information 3), we would now reclassify five of those variants as likely pathogenic (Table 2).
On the other hand, in 22 patients with succinate:fumarate ratios that were not suspicious, germline variants in $S D H x$ were identified during routine diagnostics testing. Three are known benign or likely benign $S D H x$ variants (two missense, one silent), in line with the normal succinate:fumarate ratios. However, nine patients had pathogenic or likely pathogenic germline PV, including one frameshift, one splice site, one gross deletion, and four different missense mutations already described in PPGL families. Three of those $(57,77,92)$ had tumors belonging to metabolic cluster I despite their normal succinate:fumarate. Seven of the tumors furthermore were head and neck paragangliomas, known to contain high amounts of stroma. For none of the samples was histological estimation of tumor content performed. Testing of additional tumor specimens in these patients revealed highly elevated ratios in three cases, supporting that these were false negatives due to insufficient sampling of tumor cells.

The remaining ten cases with normal succinate:fumarate had variants that would be currently classified as VUS: seven missense, one in-frame deletion, one potential splice site, and one substitution in the 5'UTR. Metabolomics analysis for additional tumor samples in eight cases confirmed normal succinate:fumarate ratios. Further analyses revealed somatic pathogenic variants in VHL and EPAS1 in two cases $(14,50)$ 


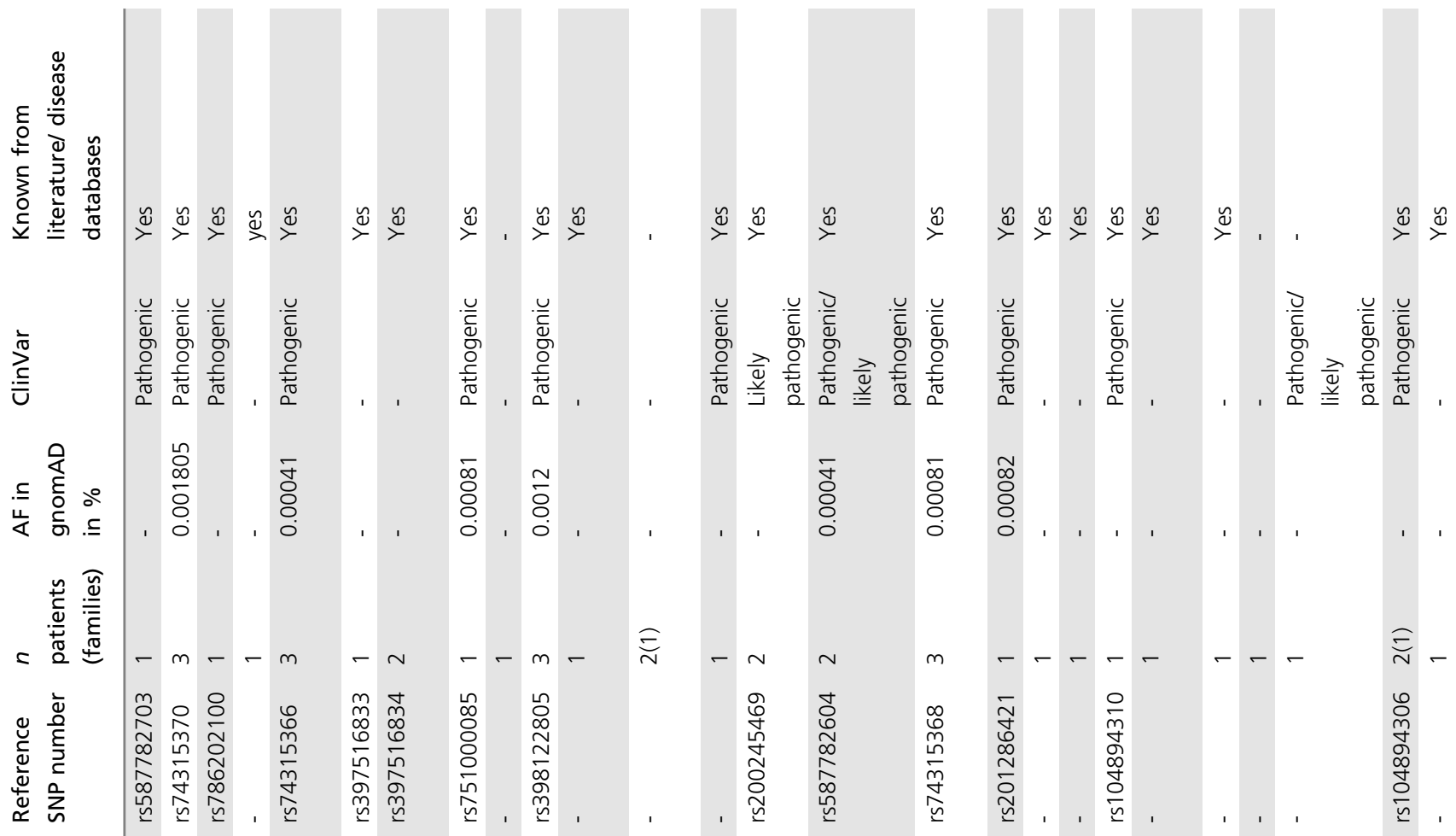

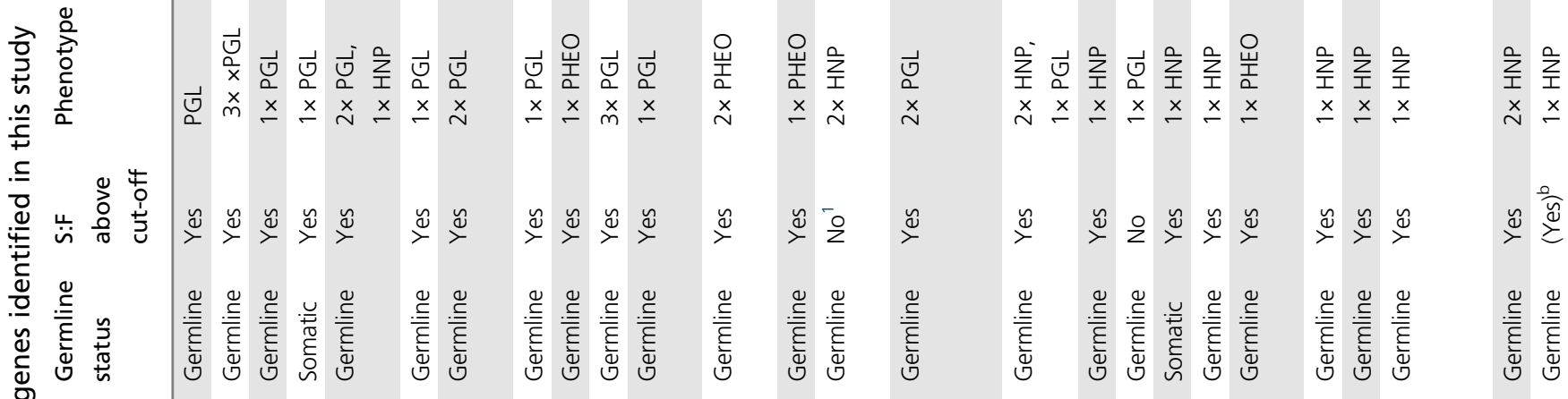

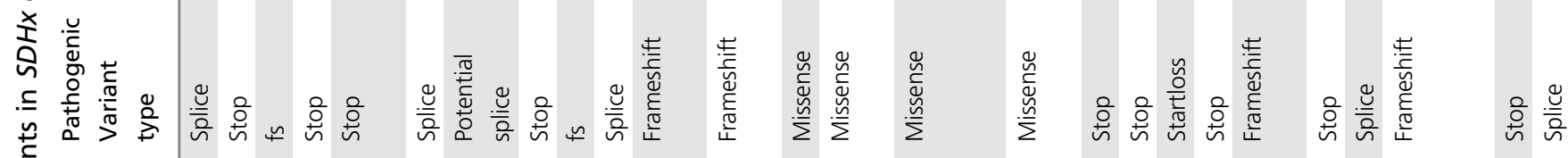

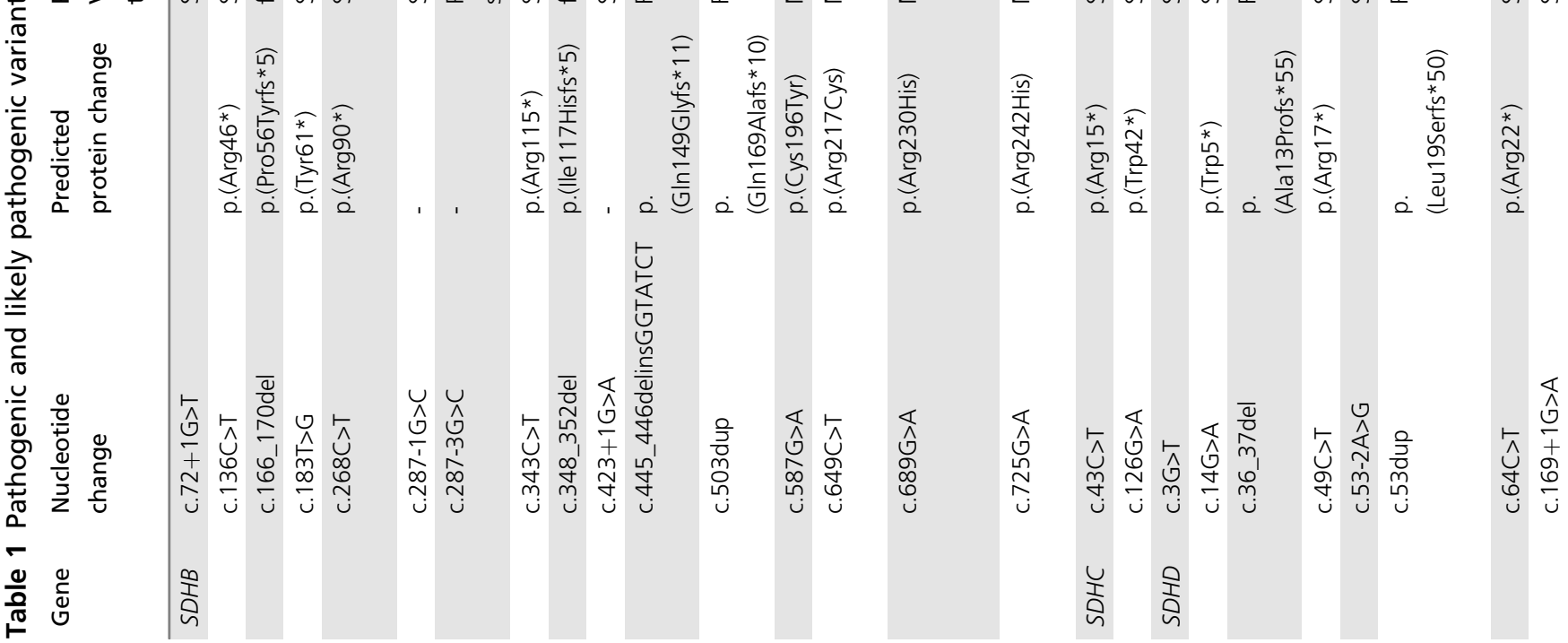




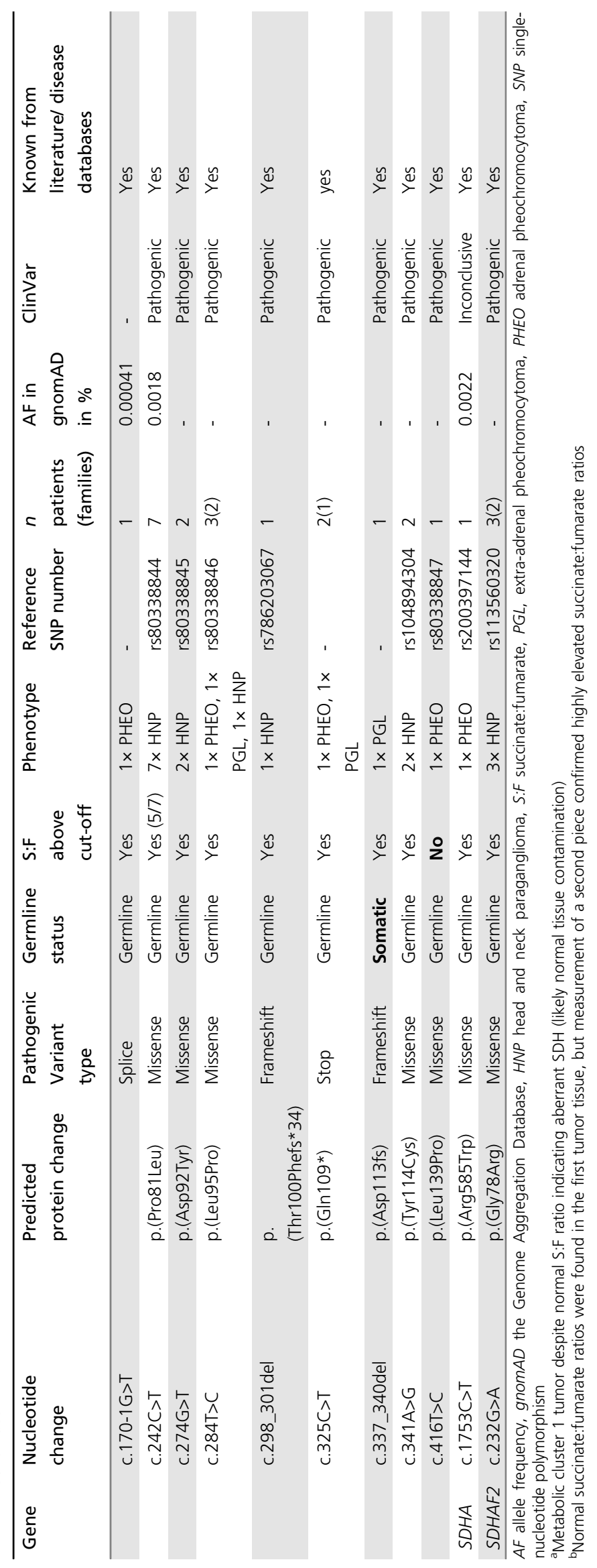




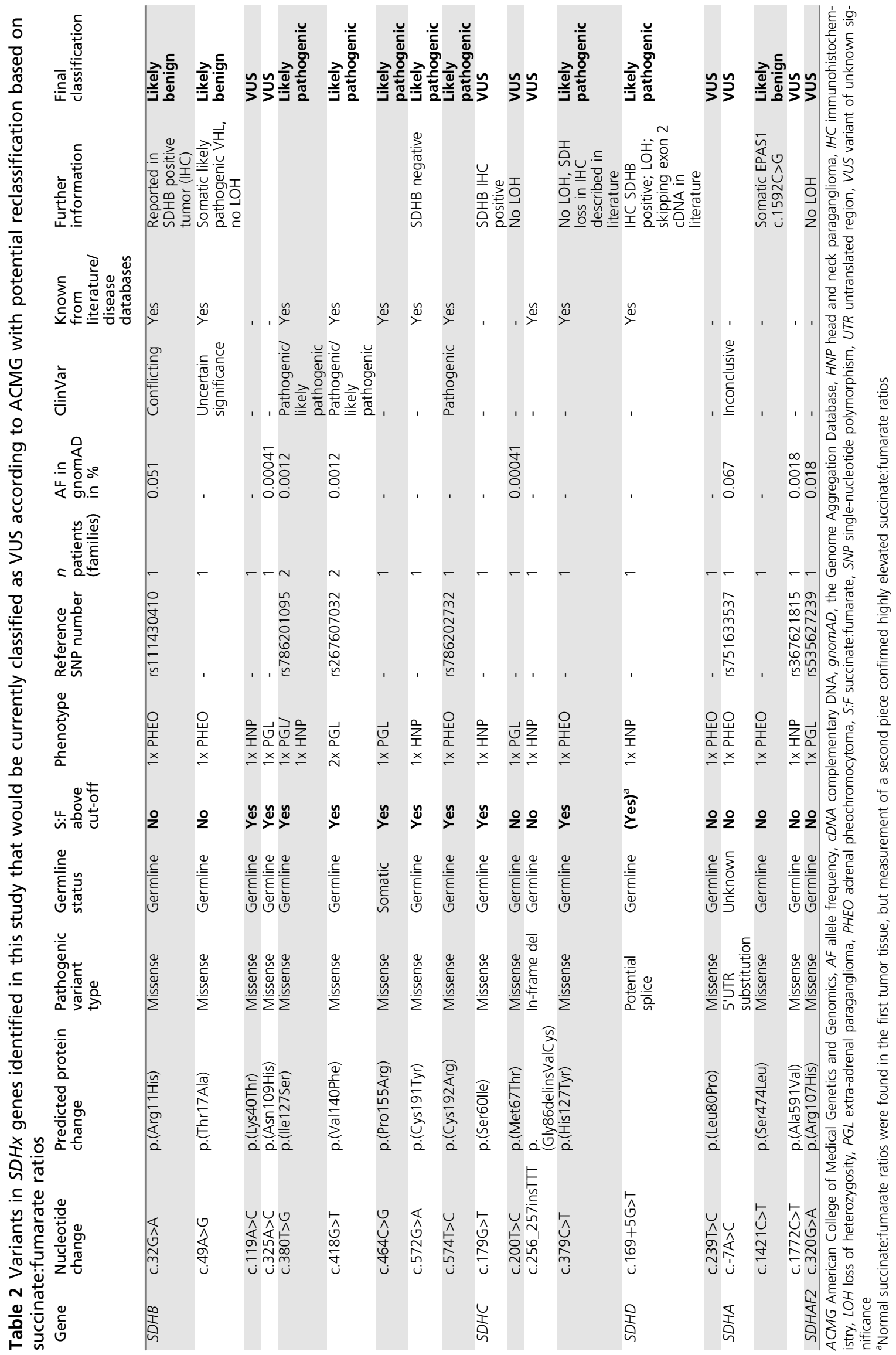


and we would reclassify the $S D H x$ variants as likely benign (Table 2). Another variant, p.(Arg11His, patient 23), has been described in an SDHB-positive tumor and together with metabolite data we would classify it as likely benign. ${ }^{35}$ In a patient (78) with a potential splice-site variant in SDHD $(c .169+5 \mathrm{G}>\mathrm{T})$ metabolomics of a second tumor specimen revealed highly elevated succinate:fumarate ratios and we would altogether reclassify this variant as likely pathogenic. For the remaining six variants, despite normal succinate: fumarate, there is currently insufficient evidence for or against pathogenicity to reclassify these variants (see Table 2 and Supplementary information 3).

Among the total of 19 VUS in SDHx identified in our cohort, subsequent to comprehensive analysis of the metabolome and the tumor genome, we would reclassify seven (37\%) as likely pathogenic and three (16\%) as likely benign (Table 2 ). The overall diagnostic performance was comparable with previously published results and is summarized in Supplementary information 4 (ref. $^{16}$ ).

\section{DISCUSSION}

Stratification of PPGL patients according to underlying $\mathrm{PV}$ is a crucial step of personalized patient management. PV in Krebs cycle-related genes are established drivers of tumor development, acting through the inhibition of $\alpha$ ketoglutarate-dependent enzymes. Identifying such driver PV has important implications for the surveillance of patients at risk of recurrence or metastasis or of carriers of germline variants who are at risk of tumor development.

Here we show that quantification of metabolites in tumor tissue can guide identification of underlying driver germline or somatic PV in patients with unresolved results of genetic testing. More specifically, characteristic metabolite profiles involving high succinate:fumarate or fumarate:malate ratios or high levels of $2 \mathrm{HG}$ in combination with high $\mathrm{D}-2 \mathrm{HG} /$ L-2HG ratios can respectively be used to guide detection of $S D H x, F H$, and IDH1/2 PV. The described mass spectrometry-based assay is simple, cheap, and uses the same instrumentation required for measurements of metanephrines, a standard test in the workup of patients with suspected PPGL. ${ }^{36}$ Metabolite data together with the catecholamine biochemical phenotype and clinical presentation can be used to narrow down the list of genes for Sanger sequencing. ${ }^{13}$ Moreover, isolated PPGL cases with rare somatic PV in IDHx or SDHx can be identified, and thereby rule out a hereditary disease in those patients.

A particular advantage of metabolome-guided genomics is that tumors with similar phenotypic presentations in terms of metabolite accumulation or depletion can be characterized, leading to identification of mechanisms of silencing known PPGL genes or even of novel susceptibility genes. Recent examples are the identification of SDHC-promoter methylation and a GOT2-activating PV in PPGL with a similar clinical presentation as in SDHx-mutated PPGLs. ${ }^{6,33}$ In our cohort, we identified five cases with somatic $S D H x$ alterations, including one SDHC-promoter methylation, all currently considered extremely rare events. ${ }^{6,33} \mathrm{We}$ also for the first time report a somatic IDH2 PVmuta in a PPGL.

The metabolome-guided identification of three patients with germline FH PV provides further information to clarify the clinical presentation of $F H$-mutated PPGLs. Consistent with the adrenal locations of tumors in our three patients, 9 of 11 previously published cases also involved adrenal tumors. $^{3,37-39}$ All three pheochromocytomas of the current study had the expected noradrenergic phenotype, but such phenotypic information has only been available for one previously described patient also with a noradrenergic tumor. Two patients under surveillance had not developed metastasis 6 or 18 years after initial diagnosis. Follow-up remains strictly recommended also due to the increased risk for RCC and leiomyomas. ${ }^{4}$

In addition to guiding genetic testing, metabolome analysis is also useful for guiding data analysis and interpretation of gene variants. The latter is particularly valuable with multigene panels that are associated with increased identification of coincidental, nonpathogenic variants. Interpretation of test results is often difficult, because many variants are uncharacterized and their diseaserelated impact remains unknown, leading to uncertainty for both patients and health-care providers. Provision of falsepositive results to patients can have significant negative consequences for patients and their families. Guidelines published by the ACMG and AMP give clear instructions on the interpretation of germline variants. ${ }^{26}$ Here we show that characterization of tumor tissue through mass spectrometry-based analysis of Krebs cycle metabolites can be employed to guide variant interpretation. When aberrant metabolomics profiling is in concordance with a VUS in a matching Krebs cycle gene, we propose that this can be used as supporting criteria for pathogenicity; if tumor metabolomics does not support a deficiency in the corresponding gene, this can be a supporting criterion against pathogenicity. Therefore, if germline genetic testing is inconspicuous or inconclusive (VUS), genetic testing of tumor tissue accompanied by metabolomics can be employed along with immunohistochemistry where appropriate. Importantly, in cases with a clear pathogenic metabolite tumor profile but without a clear pathogenic PV in the corresponding genes, alternative methods to analyze the same genes, such as promoter methylation, should be considered.

Unremarkable metabolite profiles should be interpreted in context and do not exclude a Krebs cycle gene PV ; falsenegative results can be due to high amounts of normal or stromal tissue within the sample. In line with a previous study, we found that false-negative results mainly occur in head and neck paragangliomas ${ }^{16}$ (Supplementary information 4), which may be explained by subforms with low amounts of tumor cells relative to tumor volume. ${ }^{40}$ If possible, several tissue specimens from different regions of the tumor should be investigated. Importantly, tissue specimens should be scored for tumor content by a pathologist for both metabolomics and genetic testing. 
In summary, metabolomics is a useful tool for guiding identification of $S D H x, F H$, and $I D H 1 / 2 \mathrm{PV} s$ and in variant interpretation with implications not only in PPGL, but also in other tumor diseases caused by Krebs cycle gene PV. Based on our experiences, we propose a cumulative approach for comprehensive PPGL diagnostics, including sequencing of germline and tumor tissue, as well as metabolic analyses in patients with inconclusive results of genetic testing; particularly in cases with VUS in Krebs cycle-associated genes and unsolved cases to guide diagnostic decision making. ${ }^{13}$ Our study underlines that genetic diagnostics in PPGLs is a multidisciplinary approach combining the expertise of geneticists, endocrinologists, clinical chemists, and pathologists.

\section{ELECTRONIC SUPPLEMENTARY MATERIAL}

The online version of this article (https://doi.org/10.1038/s41436018-0106-5) contains supplementary material, which is available to authorized users.

\section{ACKNOWLEDGEMENTS}

This study was funded by the Deutsche Forschungsgemeinschaft (RI 2684/1-1; KL 2541/2-1 and CRC/Transregio 205/1, B12 by B.K./G.E/J.L.), the AES PI17/01796, cofinanced by Fondo Europeo de Desarrollo Regional (FEDER), the European Union Seventh Framework Programme (FP7/2007-2013) under grant agreement no. 259735, the Paradifference Foundation, and the Intramural Research Program of the National Institutes of Health-National Institute of Child Health and Human Development.

\section{DISCLOSURE}

The authors declare that they have no conflicts of interest.

\section{REFERENCES}

1. Gaude E, Frezza C. Defects in mitochondrial metabolism and cancer. Cancer Metab. 2014;2:10.

2. Adam J, Yang $M$, Soga $T$, et al. Rare insights into cancer biology. Oncogene. 2014;33:2547-56.

3. Castro-Vega LJ, Buffet A, De Cubas AA, et al. Germline mutations in $\mathrm{FH}$ confer predisposition to malignant pheochromocytomas and paragangliomas. Hum Mol Genet. 2014;23:2440-46.

4. Schmidt LS, Linehan WM. Hereditary leiomyomatosis and renal cell carcinoma. Int J Nephrol Renov Dis. 2014;7:253-60.

5. Clark O, Yen K, Mellinghoff IK. Molecular pathways: isocitrate dehydrogenase mutations in cancer. Clin Cancer Res. 2016;22:1837-42.

6. Remacha L, Comino-Mendez I, Richter S, et al. Targeted exome sequencing of Krebs cycle genes reveals candidate cancer-predisposing mutations in pheochromocytomas and paragangliomas. Clin Cancer Res. 2017;23:6315-24.

7. Gaal J, Burnichon N, Korpershoek E, et al. Isocitrate dehydrogenase mutations are rare in pheochromocytomas and paragangliomas. J Clin Endocrinol Metab. 2010;95:1274-78.

8. Fishbein $\mathrm{L}$, Leshchiner I, Walter $\mathrm{V}$, et al. Comprehensive molecular characterization of pheochromocytoma and paraganglioma. Cancer Cell. 2017;31:181-93.

9. Xekouki P, Stratakis CA. Succinate dehydrogenase (SDHx) mutations in pituitary tumors: could this be a new role for mitochondrial complex II and/or Krebs cycle defects? Endocr Relat Cancer. 2012;19:C33-40.

10. Evenepoel L, Papathomas TG, Krol N, et al. Toward an improved definition of the genetic and tumor spectrum associated with SDH germline mutations. Genet Med. 2015;17:610-20.
11. Dahia PL. Pheochromocytoma and paraganglioma pathogenesis: learning from genetic heterogeneity. Nat Rev Cancer. 2014;14:108-19.

12. Cascon A, Comino-Mendez I, Curras-Freixes M, et al. Whole-exome sequencing identifies $\mathrm{MDH} 2$ as a new familial paraganglioma gene. J Natl Cancer Inst 2015;107 pii: djv053.

13. Eisenhofer G, Klink B, Richter $S$, et al. Metabologenomics of phaeochromocytoma and paraganglioma: an integrated approach for personalised biochemical and genetic testing. Clin Biochem Rev. 2017;38:69-100.

14. Gimenez-Roqueplo AP, Favier J, Rustin P, et al. Mutations in the SDHB gene are associated with extra-adrenal and/or malignant phaeochromocytomas. Cancer Res. 2003;63:5615-21.

15. Favier J, Amar L, Gimenez-Roqueplo AP. Paraganglioma and phaeochromocytoma: from genetics to personalized medicine. Nat Rev Endocrinol. 2015;11:101-11.

16. Richter S, Peitzsch M, Rapizzi E, et al. Krebs cycle metabolite profiling for identification and stratification of pheochromocytomas/paragangliomas due to succinate dehydrogenase deficiency. J Clin Endocrinol Metab. 2014;99:3903-11.

17. Kim E, Wright MJ, Sioson L, et al. Utility of the succinate: fumarate ratio for assessing SDH dysfunction in different tumor types. Mol Genet Metab Rep. 2017;10:45-49.

18. Pollard PJ, Briere JJ, Alam NA, et al. Accumulation of Krebs cycle intermediates and over-expression of HIF1 alpha in tumours which result from germline $\mathrm{FH}$ and $\mathrm{SDH}$ mutations. Hum $\mathrm{Mol}$ Genet. 2005; 14:2231-39.

19. Dang L, White DW, Gross S, et al. Cancer-associated IDH1 mutations produce 2-hydroxyglutarate. Nature. 2009;462:739-44.

20. Lenders JW, Duh QY, Eisenhofer G, et al. Pheochromocytoma and paraganglioma: an endocrine society clinical practice guideline. J Clin Endocrinol Metab. 2014;99:1915-1942.

21. Toledo RA, Burnichon N, Cascon A, et al. Consensus statement on nextgeneration-sequencing-based diagnostic testing of hereditary phaeochromocytomas and paragangliomas. Nat Rev Endocrinol. 2017; 13:233-47

22. Curras-Freixes M, Pineiro-Yanez E, Montero-Conde $C$, et al. PheoSeq: a targeted next-generation sequencing assay for pheochromocytoma and paraganglioma diagnostics. J Mol Diagn. 2017;19:575-88.

23. Gieldon L, Masjkur JR, Richter S, et al. Next generation panel sequencing identifies NF1 germline mutations in three patients with pheochromocytoma but no clinical diagnosis of neurofibromatosis type 1. Eur J Endocrinol. 2018;178:K1-K9.

24. Rehm HL, Bale SJ, Bayrak-Toydemir $P$, et al. ACMG clinical laboratory standards for next-generation sequencing. Genet Med. 2013;15:733-47.

25. Toledo RA, Hatakana R, Lourenco DM Jr, et al. Comprehensive assessment of the disputed RET Y791F variant shows no association with medullary thyroid carcinoma susceptibility. Endocr Relat Cancer. 2015:22:65-76

26. Richards S, Aziz N, Bale S, et al. Standards and guidelines for the interpretation of sequence variants: a joint consensus recommendation of the American College of Medical Genetics and Genomics and the Association for Molecular Pathology. Genet Med. 2015;17:405-24.

27. Flynn A, Dwight T, Harris J, et al. Pheo-Type: a diagnostic gene-expression assay for the classification of pheochromocytoma and paraganglioma. J Clin Endocrinol Metab. 2016;101:1034-43.

28. Imperiale A, Moussallieh FM, Roche $P$, et al. Metabolome profiling by HRMAS NMR spectroscopy of pheochromocytomas and paragangliomas detects SDH deficiency: clinical and pathophysiological implications. Neoplasia. 2015;17:55-65.

29. Struys EA, Jansen EE, Verhoeven NM, et al. Measurement of urinary D- and L-2-hydroxyglutarate enantiomers by stable-isotopedilution liquid chromatography-tandem mass spectrometry after derivatization with diacetyl-L-tartaric anhydride. Clin Chem. 2004;50: 1391-95.

30. Rump A, Benet-Pages A, Schubert $S$, et al. Identification and functional testing of ERCC2 mutations in a multi-national cohort of patients with familial breast and ovarian cancer. PLoS Genet. 2016;12:e1006248.

31. Eisenhofer G, Huynh TT, Pacak K, et al. Distinct gene expression profiles in norepinephrine- and epinephrine-producing hereditary and sporadic pheochromocytomas: activation of hypoxia-driven angiogenic pathways in von Hippel-Lindau syndrome. Endocr Relat Cancer. 2004;11: 897-911.

32. Allegri G, Fernandes MJ, Scalco FB, et al. Fumaric aciduria: an overview and the first Brazilian case report. J Inherit Metab Dis. 2010:33:411-19. 
33. Richter S, Klink B, Nacke B, et al. Epigenetic mutation of the succinate dehydrogenase $C$ promoter in a patient with two paragangliomas. J Clin Endocrinol Metab. 2016:101:359-63.

34. Cascon A, Ruiz-Llorente S, Cebrian A, et al. G12S and H50R variations are polymorphisms in the SDHD gene. Genes Chromosomes Cancer. 2003;37:220-21.

35. van Nederveen FH, Gaal J, Favier J, et al. An immunohistochemical procedure to detect patients with paraganglioma and phaeochromocytoma with germline SDHB, SDHC, or SDHD gene mutations: a retrospective and prospective analysis. Lancet Oncol. 2009;10:764-71.

36. Peitzsch M, Pelzel D, Glockner $S$, et al. Simultaneous liquid chromatography tandem mass spectrometric determination of urinary free metanephrines and catecholamines, with comparisons of free and deconjugated metabolites. Clin Chim Acta. 2013;418:50-58.

37. Letouze $E$, Martinelli $C$, Loriot $C$, et al. SDH mutations establish a hypermethylator phenotype in paraganglioma. Cancer Cell. 2013;23:739-52.

38. Clark GR, Sciacovelli M, Gaude E, et al. Germline FH mutations presenting with pheochromocytoma. J Clin Endocrinol Metab. 2014;99: E2046-50.

39. Nambuba J, Därr R, Janssen I, et al. Functional imaging experience in a germline fumarate hydratase mutation-positive patient with pheochromocytoma and paraganglioma. Clin Case Rep. 2016;2:e176-81.

40. Williams MD. Paragangliomas of the head and neck: an overview from diagnosis to genetics. Head Neck Pathol. 2017;11:278-87. 